\title{
Research on Characteristics of Nonlinear Analog Devices Based on RBF Neural Network
}

\author{
Yiwei Liu \\ North China Electric Power University, Changing Destrict, Beijing, P.R.China,102206 \\ Yiwei.liu@ncepu.edu.cn
}

Keywords: Artificial Neural Network; Radial Basis Function; Tunnel Diodes; Volt-ampere Characteristics

\begin{abstract}
Radial basis function (RBF) neural network is a novel and effective feedforward neural network which has been widely used in nonlinear time series prediction. In this paper, the tunnel diode is selected as a modeling object to measure the input and output characteristics in the laboratory environment. We analyze the necessity and feasibility of this modeling method in the actual circuit design, and can use the EDA tool to design the component model independently, and give us a more valuable circuit simulation before the analog circuit design.
\end{abstract}

\section{Introduction}

In the context of analog circuits, most of the components we face are non-linear. With the rapid development of modern EDA tools, computer simulation technology for analog and analog circuits has brought great convenience to electronic designers. But the characteristics of these analog components are still constrained by its production process, application environment and other objective factors. Therefore, the computer-aided analysis of an analog component often falls under its optimal conditions which is different form the analysis in practice.

Therefore, it is necessary to propose a mean of stimulating the characteristics of analog and analog system based on the measured data. With the rapid development of modern intelligent algorithms, a large number of methods for nonlinear data fitting simulation have emerged. Neural network is one of the most representative methods.

\section{Introduction to Neural Network}

\section{Introduction to Artificial Neural Networks}

Artificial neural network refer to the network widely interconnected by a large number of processing units. As the abstraction, simplification and simulation of the human brain, it can reflect the basic characteristics of the human brain. In general, the artificial neural network should have three elements.

(1) Has a set of prominent or joins, we generally use $\mathbf{w}_{i j}$ to represent the junction strength between neurons $\mathbf{i}$ and $\mathbf{j}$ recorded as weight. The weight value of artificial neurons can be positive or negative.

(2) Has an input signal accumulator that reflects the spatial and temporal integration of biological neurons;

(3) Has an excitation function to limit the output of neurons. The excitation function compresses the output signal within an allowable range. 


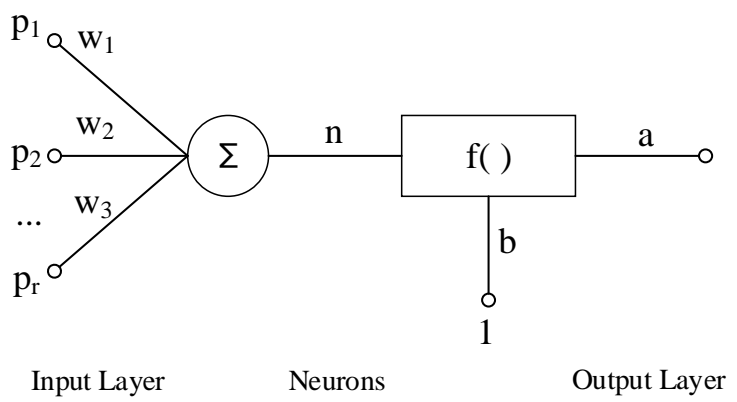

Figure 1. Artificial neural network model

A neuron structure simulation with $\mathbf{r}$ input classification is shown in Figure 1. Its most important part is the activation function $\left.{ }^{\mathrm{f}(}\right)$, which generally has two inputs:

(1) The input component $\mathbf{p}_{\mathbf{j}}(\mathbf{j}=1,2, \ldots, \mathbf{r})$ is connected by the weight component $\mathbf{w}_{\mathbf{j}}(\mathbf{j}=1,2, \ldots, \mathbf{r})$ multiplied by it, and summed with $\stackrel{\mathrm{j}}{\mathrm{j}=1}^{\mathrm{r}} \mathbf{w}_{\mathrm{j}} \mathbf{p}_{\mathrm{j}}$ as the input of the activation function ${ }^{\mathbf{f}(x)}$;

(2) The deviation between the neuron output and the expected $\mathbf{b}$ input is regarded as the input of an activation function.

Activation function generally has three options as shown in Table 1:

Table 1 Common activation function

\begin{tabular}{|c|c|}
\hline Function name & Function expression \\
\hline Threshold function & $f(x)=\begin{array}{l}\frac{1}{1} 1, x^{3} \quad c \\
10, x<c\end{array}$ \\
\hline Piecewise linear function & 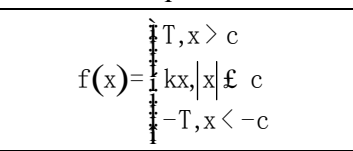 \\
\hline Sigmoid function & $f(x)=\frac{1}{1+e^{-\alpha x}}(0<f(x)<1)$ \\
\hline
\end{tabular}

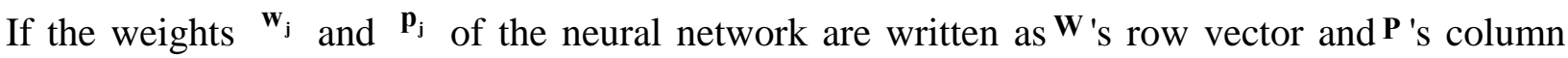
vector representation:

$$
\mathbf{W}=\left[\mathbf{w}_{1}, \mathbf{w}_{2}, \ldots, \mathbf{w}_{\mathbf{r}}\right] \quad \mathbf{P}=\left[\mathbf{p}_{1}, \mathbf{p}_{2}, \ldots, \mathbf{p}_{\mathbf{r}}\right]^{\mathrm{T}}
$$

The output vector can be written as:

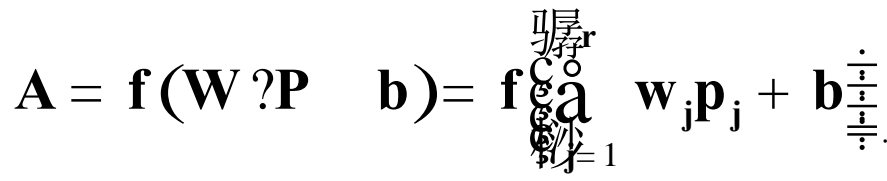

The iterations are constantly changing that allow the activation function to move continuously to increase the likelihood of finding a solution to the problem.

\section{Introduction to RBF Neural Network Model}

As with the general neural network model, RBF neural network structure includes input layer, hidden layer and output layer. The input layer directly maps the input vector to the hidden space and plays the role of transmitting signal. The hidden layer contains a number of hidden cells. The hidden layer can be mapped by the activation function. The activation function is a radial symmetry, The non-negative nonlinear function of the attenuation; the output layer is a mapping of the linear weighted sum of the hidden layer.

Linearity and non-linearity are combined by RBF neural network. From input layer to implicit 
layer, non-linear mapping form and nonlinear optimization strategy are adopted that can not be quickly learned. Linear transformation and linear optimization strategy are adopted from linear layer to output layer that can be quickly learned.

Input layer Implicit layer Output layer

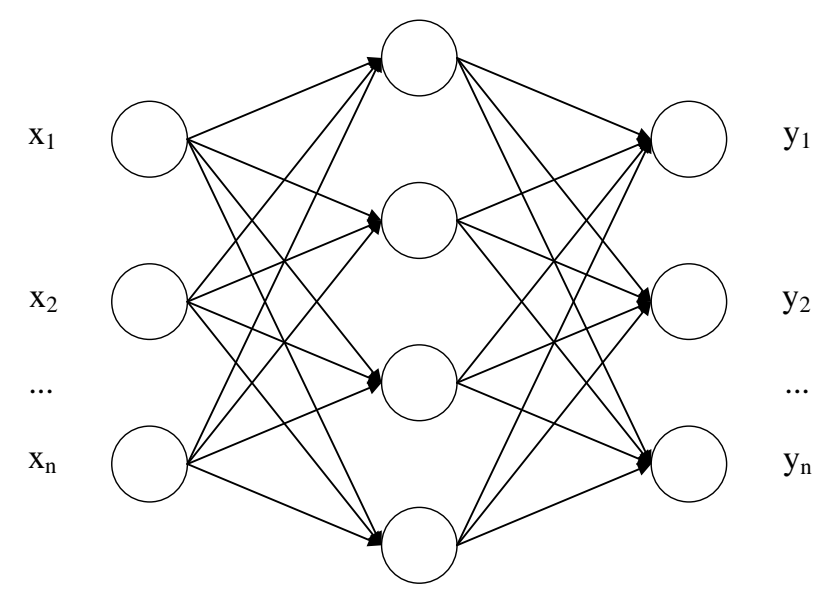

Figure 2. The architecture of RBF neural network

The activation function of the implicit layer of RBF neural network is generally defined as a monotonic function of Euclidean distance between any point in space, for example, Gaussian function ${ }^{[2]}$ :

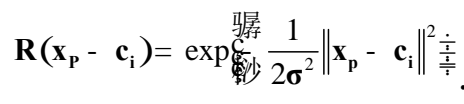

In this formula: $\|_{\text {is the European norm, }} \mathbf{c}$ is the center of the Gaussian function, and $\boldsymbol{\sigma}$ is the variance of the Gaussian function. The output of the RBF network model is weighted by the output of the hidden layer neurons, and the output is ${ }^{[2]}$ :

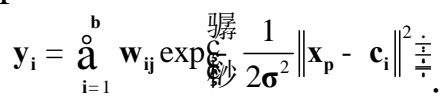

In this formula: $\mathbf{x}_{\mathbf{p}}$ is the $\mathbf{p}(\mathbf{p}=1,2,3, \ldots, \mathbf{P})$ th input sample, $\mathbf{c}_{\mathbf{i}}$ is the center of the implied node layer; $\mathbf{w}_{\mathbf{i j}}$ is the connection weight of the hidden layer to the output layer; $\mathbf{i}=1,2,3, \ldots, \mathbf{h}$ represents the total of $\mathbf{h}$ nodes, and $\mathbf{j}=1,2,3, \ldots, \mathbf{n}$ represents the total $\mathbf{n}$ output nodes; $\mathbf{y}_{\mathbf{i}}$ represents the actual output of the $\mathbf{j}_{\text {th }}$ output node of the network corresponding to the input sample.

RBF network learning also requires parameters include the center of the basis function, variance and weight. We generally select $\mathbf{c}$ as the base function center and then solve the variance $\boldsymbol{\sigma}$. Based on the Gaussian function, the variance of the basis function is:

$$
\boldsymbol{\sigma}_{\mathrm{i}}=\frac{\mathbf{c}_{\max }}{\sqrt{2 \mathbf{h}}}
$$

In this formula: $\boldsymbol{c}_{\max }$ are the maximum distance of the selected center, $\mathbf{i}=1,2,3, \ldots, \mathbf{h}$.

The weight of the neuron connection between the hidden and output layers can be calculated with the least squares method.

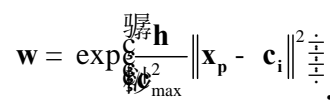

The parameters of the center and weight are gradually adjusted by RBF neural network according to the relationship of the input error and the output error, and by which the internal coefficients of the whole network are adjusted. The termination of network calculation and the output of the prediction value are not carried out until the network mean square error reaches the default accuracy range by the repeated iterative calculation. 


\section{Matlab Neural Network Toolbox}

As a mathematical application software most widely used, Matlab provides us with a neural network toolbox. We can use this toolbox to simulate and train the neural network model to be built, and we can understand the dynamic training process by changing the graph.

In the neural network toolbox, we can use the newrb( ) function to design the RBF network to add hidden nodes to the hidden layer, the call format is:

net $=$ newrb (P, T, goal, spread, MN, DF)

\section{Description}

(1) where $\mathrm{P}$ and $\mathrm{T}$ represent the input sample vector and the output target vector respectively; where $\mathrm{P}$ is the matrix of $\mathrm{R} \times \mathrm{Q}$, and $\mathrm{T}$ is the expected output matrix of $\mathrm{S} \times \mathrm{Q}$; where $\mathrm{R}$ is the input vector dimension, and $\mathrm{S}$ is the output node Number (output vector dimension).

(2) Goal is the set target mean square error network;

(3) Spread is the diffusion rate of the radial basis function, the default value is 1 ;

(4) MN is the maximum number of hidden nodes, the default value is Q;

(5) The DF indicates the number of neurons added between the two displays, which is a parameter that controls the display level. The default value is 25 .

In this function, RBF network is designed with the iterative method, a neuron is added by an iteration, iteration is not stoped until the mean square error falls to the target error or the maximum number of neurons reaches the maximum ${ }^{[3]-[4]}$.

In Matlab, we use the function sim () to simulate the built-in neural network, following is the call format of function.

$[Y$, Pf, Af, E, perf $]=\operatorname{sim}($ net, $P, P i, A i, T)$

In which, net is the object of neural network; $\mathrm{P}$ is the network input; $\mathrm{Pi}$ is the initial state of the input delay; $\mathrm{Ai}$ is the initial state of the layer delay; $\mathrm{T}$ is the target vector. In the function return value, $\mathrm{Y}$ is the network output; $\mathrm{Pf}$ is the input delay state when the training is terminated; Af is the layer delay state at the end of the training; $\mathrm{E}$ is the error between the output and the target vector; perf is the performance value of the network.

\section{Application Case}

\section{Device Selection}

Tunnel diodes are chosen by us as a case of applying study of RBF neural network characteristics. Tunnel diodes are semiconductor devices based on heavily doped PN junction tunneling. Its forward current-voltage relationship shows negative resistance characteristics, and commonly used in high-frequency circuit.

Tunnel diodes have the characteristics of high operating frequency, low cost, fast input response, high reliability, low power consumption and low noise which can be used for microwave mixing, detection, low noise amplification circuit design. In addition, the tunnel diodes also are widely used in the satellite microwave equipment, can which can also be used for the designs of ultra-high-speed switching logic circuits, flip-flops and memory circuit. In a word, it is a semiconductor device with wide application $^{[5]}$.

\section{Modeling Process}

A tunnel diode terminal voltage and the diode current data flow through the tunnel diode can be got by measurement which are shown in Table $2^{[6]-[8] \text {. }}$ 
Table 2. Test value of voltage and current in a tunnel diode

\begin{tabular}{c|c|c|c|c|c|c|c|c}
\hline Ordinal & $\mathbf{U}(\mathbf{V})$ & $\mathbf{I}(\mathbf{m A})$ & Ordinal & $\mathbf{U}(\mathbf{V})$ & $\mathbf{I}(\mathbf{m A})$ & Ordinal & $\mathbf{U}(\mathbf{V})$ & $\mathbf{I}(\mathbf{m A})$ \\
\hline 1 & 0 & 0 & 10 & 0.18 & 1.308 & 19 & 0.36 & 0.3787 \\
\hline 2 & 0.02 & 2.696 & 11 & 0.2 & 1.031 & 20 & 0.38 & 0.3749 \\
\hline 3 & 0.04 & 4.104 & 12 & 0.22 & 0.8374 & 21 & 0.4 & 0.4130 \\
\hline 4 & 0.06 & 4.601 & 13 & 0.24 & 0.7536 & 22 & 0.42 & 0.5051 \\
\hline 5 & 0.08 & 4.475 & 14 & 0.26 & 0.6569 & 23 & 0.44 & 0.6378 \\
\hline 6 & 0.1 & 3.860 & 15 & 0.28 & 0.5720 & 24 & 0.46 & 0.7931 \\
\hline 7 & 0.12 & 3.071 & 16 & 0.3 & 0.5016 & 25 & 0.48 & 1.138 \\
\hline 8 & 0.14 & 2.319 & 17 & 0.32 & 0.4468 & 26 & 0.5 & 2.610 \\
\hline 9 & 0.16 & 1.710 & 18 & 0.34 & 0.4049 & 27 & 0.52 & 8.013 \\
\hline
\end{tabular}

For convenience of our verification, the measured data in the serial number of the input and output characteristics of the data are selected by us shown in Table 3:

Table 3. Select the following set of test values to train the RBF network

\begin{tabular}{c|c|c|c|c|c|c|c|c}
\hline Ordinal & $\mathbf{U}(\mathbf{V})$ & $\mathbf{I}(\mathbf{m A})$ & Ordinal & $\mathbf{U}(\mathbf{V})$ & $\mathbf{I}(\mathbf{m A})$ & Ordinal & $\mathbf{U}(\mathbf{V})$ & $\mathbf{I}(\mathbf{m A})$ \\
\hline 1 & 0 & 0 & 6 & 0.2 & 1.031 & 11 & 0.44 & 0.6378 \\
\hline 2 & 0.04 & 4.104 & 7 & 0.24 & 0.7536 & 12 & 0.48 & 1.138 \\
\hline 3 & 0.08 & 4.475 & 8 & 0.28 & 0.572 & 13 & 0.52 & 8.013 \\
\hline 4 & 0.12 & 3.071 & 9 & 0.32 & 0.4468 & 14 & 0.4 & 0.413 \\
\hline 5 & 0.16 & 1.71 & 10 & 0.36 & 0.3787 & \multicolumn{1}{|l}{} \\
\hline
\end{tabular}

RBF network is established by us based on the results shown in Table 3 to carry out the characteristics of approximation. After the simulation experiment, when the number of neurons in the hidden layer reaches 14, the error of the input and output characteristic curve of the tunnel diode can reach the error range (error-goal <0.0019), and the error performance curve is shown in Fig. 3. It is not difficult to be found that the sum of squares of errors is gradually reduced until the set target is reached. The error square sum of 0.0019927 is got after the completion of training which can meet the set requirements. The weights and thresholds obtained are got after recording training shown in Table 4.

Table 4. Weight and threshold of RBF network

\begin{tabular}{c|c|c|c|c}
\hline $\boldsymbol{n}$ & $\boldsymbol{C}_{\boldsymbol{n}}$ & $\boldsymbol{B}_{\boldsymbol{n}}$ & $\boldsymbol{W}_{\boldsymbol{n}}$ & $\boldsymbol{B}_{\boldsymbol{o}}$ \\
\hline 1 & 0.2800 & 1.1537 & -0.154 & 1.599 \\
\hline 2 & 0.5200 & 1.5137 & -1.335 & \\
\hline 3 & 0.4800 & 1.5137 & 2.469 & \\
\hline 5 & 0 & 1.5137 & -5.003 & 15.751 \\
\hline 6 & 0.0400 & 1.5137 & 0 & \\
\hline 7 & 0.4400 & 1.5137 & 0 & \\
\hline 1 & 0.4000 & 1.5137 & -2.424 & \\
\hline 11 & 0.3600 & 1.5137 & -13.794 & \\
\hline 12 & 0.0800 & 1.5137 & 0 & \\
\hline 13 & 0.3200 & 1.5137 & 0 & \\
\hline 14 & 0.1600 & 1.5137 & 0 & \\
\hline
\end{tabular}

The error performance curve is shown in Figure 3: 


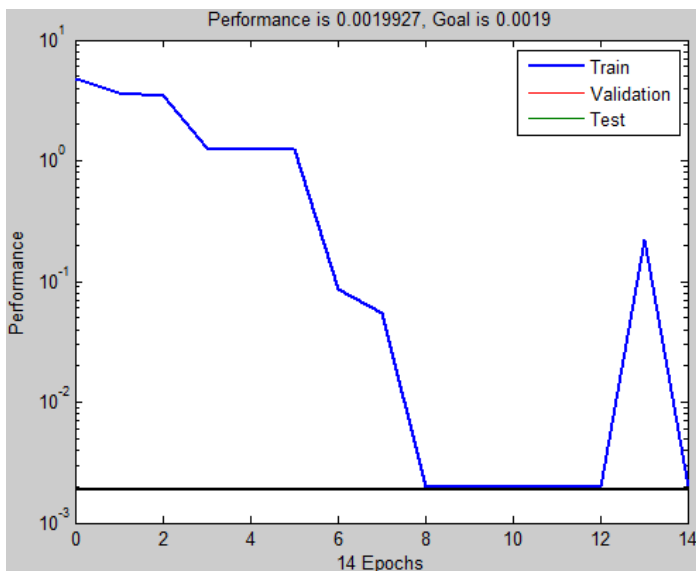

Figure 3. The training error of RBF neural network

In order to verify the accuracy of the resulting curve, the even-numbered values of the measured values are shown in Table 5:

Table 5. Select the test results to verify the simulation results.

\begin{tabular}{|c|c|c|c|c|c|c|c|c|}
\hline Ordinal & $\mathrm{U}(\mathrm{V})$ & $\mathrm{I}(\mathrm{mA})$ & Ordinal & $\mathrm{U}(\mathrm{V})$ & $\mathrm{I}(\mathrm{mA})$ & Ordinal & $\mathrm{U}(\mathrm{V})$ & $\mathrm{I}(\mathrm{mA})$ \\
\hline 1 & 0.02 & 2.696 & 6 & 0.22 & 0.8374 & 11 & 0.42 & 0.5051 \\
\hline 2 & 0.06 & 4.601 & 7 & 0.26 & 0.6569 & 12 & 0.46 & 0.7931 \\
\hline 3 & 0.1 & 3.86 & 8 & 0.3 & 0.5016 & 13 & 0.5 & 2.61 \\
\hline 4 & 0.14 & 2.319 & 9 & 0.34 & 0.4049 & & & \\
\hline 5 & 0.18 & 1.308 & 10 & 0.38 & 0.3749 & & & \\
\hline
\end{tabular}

They are placed in the fitted characteristic curve, as shown in Figure 4:

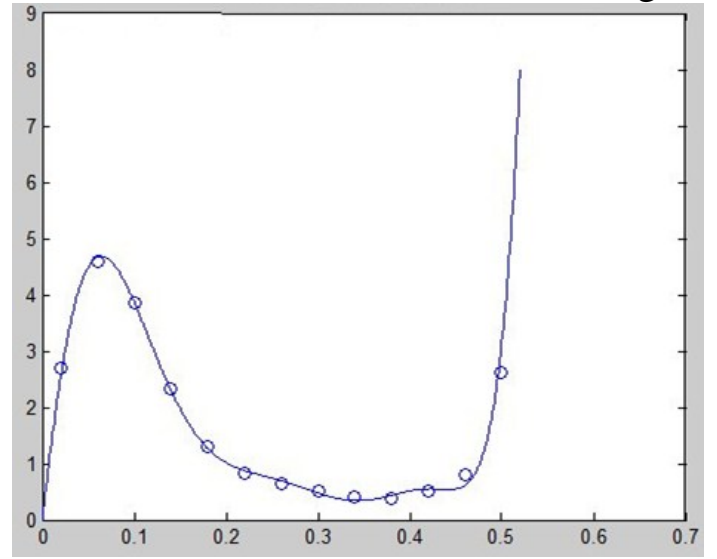

Figure 4. The consequence of curve fitting

As shown in Fig. 4, the solid line is the fitting curve obtained by successive approximation with RBF neural network. The dots represent the voltammetric characteristics of the measured tunnel diodes in some positions.

According to the fitting curve shown in Fig. 4, it is not difficult to be found that the effect of curve fitting basically satisfies the requirement. Similarly, according to the error curve shown in Fig. 3 , it can be found that the error gradually converges to meet the requirements of our setting. Therefore, the nonlinear characteristic curve fitting method of tunnel diode based on RBF network is successful.

\section{Summary}

There are a lot of non-linear components in Analog circuits, and the input and output characteristics of these components are affected by many external factors such as the manufacturing process and 
temperature. We must consider these external factors in designing the circuit. However, in the environment of simulation software, it is difficult to ensure that the manufacturing process and temperature of the device are consistent with the actual circuit. At this time, we can actually build the measurement circuit around these devices to carry out the approximation of the input and output of our target with RBF neural network. The actual non-linear device model can be built with this method.

In fact, it is reported in some literature that Pspice can be used to build the device model for RBF neural network ${ }^{[9]}$. The corresponding center, weight and threshold can be got with the help of the training network by inputting the input and output data of component modeled by computer. In other word, the model of the component can be built in Pspice. RBF neural network learning has high speed and precision, this modeling is simple and easy way, and which can be extended to more components such as regulator and rectifier.

Based on the above analysis, we can model some complex devices such as transistors, FET and other multi-input devices. In addition, we can also model some packaged analog electronic system to get the total input and output characteristics of a system. In short, there are many tasks needed to be done by us, we should carry out more modelings for more devices with the help of RBF network.

\section{References}

[1] Huang Ying. Neural network based on the nonlinear electronic device modeling method [D]. Changchun: Northeast Normal University, 2007;

[2] Zhang Xiaolin; Wang Zhenbo, et al.Comparative Analysis of Urban Built-up Area and BP Neural Network and Linear Regression Based on RBF Neural Network [J]. Journal of Yangtze River Basin Resources and Environment, 2013 (22-6): 691-697.

[3] Chen Ming. Matlab neural network principle and application [M]. Beijing: Tsinghua University Press, 2015: 475-481.

[4] Pu Haiming.Study on optimization method based on neural network nonlinear electronic device modeling [D]. Changchun: Northeast Normal University, 2007;

[5] Sun Huimin. Tunnel diode manufacturing and application [J]. Source is unknown;

[6] JOHN H, ANDERS K. Non-linear back -propagation: doing without derivatives of the activation function[J]. IEEE Transactions on Neural Networks, 1997 (8-6) : 1321 -1327.

[7] Sheng Zhongping, Wang Xiaohui, Sun Xuenan. Multi-point multiple Newton interpolation formula [J]. Journal of Northeast Normal University: Natural Science Edition, 2007, (40-4): $136-137$.

[8] Liu Heng, Jiang Fengyi, Xia Bin, et al.Experimental study of multi-scroll chaotic circuit based on analog inductor [J]. Journal of Northeast Normal University: Natural Science Edition, 2007 (40-2): 55-59.

[9] Wang Lianming, Huang Ying.Study on modeling method of nonlinear electronic device based on RBF neural network [J]. Journal of Northeast Normal University: Natural Science Edition, 2008 (40-3): 46-50. 